\title{
Lidocaine Transdermal Patch: Pharmacokinetic Modeling and In Vitro-In Vivo Correlation (IVIVC)
}

\author{
Phani Krishna Kondamudi, ${ }^{1,5}$ Phani Prasanth Tirumalasetty, ${ }^{2,5}$ Rajkumar Malayandi, ${ }^{1,5}$ \\ Srinivas Mutalik, ${ }^{3}$ and Raviraj Pillai ${ }^{4,5,6}$
}

Received 16 June 2015; accepted 5 August 2015; published online 18 August 2015

\begin{abstract}
The present study aims to develop the correlation between in vitro and in vivo skin permeation of lidocaine in its transdermal patch. In order to minimize the run-to-run variability during in vitro skin permeation studies, release normalized cumulative percent $\left(\% C t_{n}\right)$ was calculated. A suitable polynomial mathematical model was used to establish a correlation between time and $\% C t_{\mathrm{n}}$. Percent in vivo absorbed was calculated by using numerical deconvolution (NDC) and non-compartmental analysis (NCA) methods. Pharmacokinetic (PK) parameters such as $A U C_{\text {last }}$ and $C_{\max }$ were predicted with the established in vitro-in vivo correlation (IVIVC) models. The minimum prediction errors in NDC method for $C_{\max }$ were found to be -30.9 and $-25.4 \%$ for studies I (in vivo study in human volunteers with one batch of Lidoderm patch; internal validation) and II (in vivo study in human volunteers with another batch of Lidoderm patch; external validation), respectively, whereas minimum prediction errors in NCA method were relatively low (3.9 and $0.03 \%$ for studies I and II, respectively) compared to those in NDC method. The prediction errors for $A U C_{\text {last }}$ were found to be less than $2 \%$ for both methods and studies. The established method in this study could be a potential approach for predicting the bioavailability and/or bioequivalence for transdermal drug delivery systems.
\end{abstract}

KEY WORDS: bioequivalence; IVIVC; lidocaine; numerical deconvolution; transdermal.

\section{INTRODUCTION}

Transdermal drug delivery systems are generally used to achieve entry of drugs directly into systemic circulation, bypassing the first-pass metabolism, for the desired therapeutic effect. In support of this, very few transdermal

Phani Krishna Kondamudi is a Scientist at the Centre of Excellence, Bio-Studies; Phani Prasanth is a Principal Scientist at the Formulation Research \& Development, Softgel, Topical, Transdermal and Respiratory (STaR) Vertical; Rajkumar Malayandi is a Senior scientist at the Centre of Excellence, Bio-Studies; Srinivas Mutalik is a Professor at the Department of Pharmaceutics, Manipal College of Pharmaceutical Sciences; Raviraj Pillai is the Vice-President and Delivery Head of Product Development at the Integrated Product Development Organization, Dr. Reddy's Laboratories.

${ }^{1}$ Centre of Excellence- Bio Studies, Hyderabad, Telangana, India.

${ }^{2}$ Formulations R \& D, Softgel, Topical, Transdermal and Respiratory (STaR) Vertical, Hyderabad, India.

${ }^{3}$ Department of Pharmaceutics, Manipal College of Pharmaceutical Sciences, Manipal, 576104, Karnataka, India.

${ }^{4}$ Softgel-Transdermal/Topical-Respiratory (STaR) Product Development, Dr.Reddy's Laboratories, Hyderabad, 500090, Andhra Pradesh, India.

${ }^{5}$ Integrated Product Development Organisation, IPDO- Innovation Plaza, Dr. Reddy's Laboratories, Bachupally, R R District, Hyderabad, 500090, India.

${ }^{6}$ To whom correspondence should be addressed. (e-mail: ravip@drreddys.com) patches have been developed and marketed for local therapy (1). Most of the transdermal patches intended for local therapy exhibit plasma drug concentrations below the limit of quantification and hence pose low risk for systemic toxicities. Lidocaine topical patch (LTP) containing 5\% $w / w$ of lidocaine is approved by the USFDA under the brand name of Lidoderm ${ }^{\circledR}$ [reference listed drug (RLD)] for relief of pain associated with post-herpetic neuralgia (2). The systemic bioavailability (BA) of LTP is $3 \pm 2 \%$ (3). The maximum plasma drug concentration achieved is $\sim 45 \mathrm{ng} / \mathrm{mL}$ after application of three patches simultaneously for the period of $12 \mathrm{~h}$, whereas the tolerable plasma drug concentration is 1500 to $5000 \mathrm{ng} / \mathrm{mL}$ for the treatment of cardiac arrhythmia (4). Hence, there is limited role of plasma drug concentration with respect to localized analgesic effect of LTP. Following administration of LTP, lidocaine produces local analgesic effect without complete sensory block (5). Even though evaluation of plasma drug concentration is not an accurate measure for drug availability at the site of action for topical products, in the absence of well-defined clinical methodology, bioequivalence (BE) with pharmacokinetic (PK) end point serves as a surrogate for establishing the equivalence between generic and branded LTP (6). The published draft guidance on Lidoderm ${ }^{\circledR}$ (RLD; non-binding) recommends the bioequivalence (BE) study along with adhesion test, skin irritation, and sensitization test to prove similarity of the generic LTP with the RLD (7). In response to Endo 
Pharma's Citizen Petition, FDA recommended (6) the following factors to be considered for the conduct of pharmacokinetic study:

(a) Lidocaine can be measured in plasma following its application onto the skin.

(b) Lidocaine is expected to pass through the stratum corneum to reach its site of action.

(c) Capillaries are present throughout dermal tissue beneath the stratum corneum, where lidocaine is expected to enter the bloodstream through these capillaries.

(d) Lidocaine is expected to be present in plasma at a concentration proportional to its presence at the site of action, when applied topically on to the skin.

The adverse drug reactions of LTP in severe cardiac impairment patients are due to the elevated plasma drug concentration. Moreover, lidocaine metabolites are known to be genotoxic, carcinogenic, and mutagenic (8). Generic development of transdermal patches is a challenging task because of various technical, intellectual property (IP), and regulatory issues. Establishing bioequivalence between RLD and test products is cumbersome due to low systemic bioavailability of LTP, when compared with other dosage forms. The reasons for bioinequivalence between test product and RLD might be the differences in qualitative and quantitative composition and the pharmaceutical processes adopted for the manufacture of these patches. Like other dosage forms, in vitro drug release testing (IVRT) methodologies are traditionally used for the optimization of formula and process for transdermal patches. Various IVRT methodologies are recommended by the Office of Generic Drugs (OGD), which include use of United States Pharmacopoeia (USP) apparatus 5 (paddle over disk method), USP apparatus 6 (rotating cylinder method), and USP apparatus 7 (reciprocating holder method) for topical and transdermal products (9). Vertical diffusion cell method has been adopted by USP to evaluate semisolid and transdermal dosage forms (10). Traditionally, these methods are used as quality control tools. To date, very few attempts have been made to develop biorelevant IVRT methods and to establish in vitro-in vivo correlation (IVIVC) for transdermal patches (11-15). The traditional IVIVC methods using PK model and numerical deconvolution (NDC) method have their own limitations such as requirement of intravenous (IV) data, unit impulse response (UIR) values, and errors associated with model building (16). Non-compartmental analysis (NCA) has been widely accepted by regulatory agencies to compare the pharmacokinetic data between test and RLD products to evaluate bioequivalence (17). In the present method, we have attempted to develop an NCA-based IVIVC model and then to compare the results with IVIVC model developed by NDC. The identified method(s) may be used as a biorelevant quality control (BRQC) in vitro tool for pharmaceutical quality assurance.

\section{MATERIALS AND METHODS}

\section{Materials}

Lidoderm $^{\circledR}$ patch (Endo Pharmaceuticals, USA; Batch No. 13248 for study I and Batch No. Z0248 for study II) was obtained from the local pharmacy from the USA. Internal
Standard (Lidoacine-d10; Lot No. H154P19) was procured from $\mathrm{C} / \mathrm{D} / \mathrm{N}$ Isotopes Inc., Canada. All the reagents used for estimation of lidocaine were of analytical grade.

\section{Ex Vivo Skin Permeation Studies}

Franz diffusion cells were used to investigate the ex vivo skin permeation of lidocaine from transdermal patches. Human cadaver skin (HCS) was used as the barrier (18). A $0.64-\mathrm{cm}^{2}$ patch containing $3.2 \mathrm{mg}$ of drug was placed on the surface of the HCS. Physiological parameters of the skin such as age and sex of the donor, skin cultivation site, transepidermal water loss, thickness of skin, and preparation method of the skin play an important role in in vitro skin permeation studies (19). Hence, these parameters were carefully monitored, controlled, and captured in the study protocol. The study protocol was approved by investigative review board. The temperature at which the study was performed is $37 \pm 2^{\circ} \mathrm{C}$. Preparation of skin for ex vivo permeation study has been performed by thawing the skin in $0.9 \% \mathrm{NaCl}$ for not more than $1 \mathrm{~h}$ at room temperature and cutting to appropriate Franz cell size $\left(\approx 5 \mathrm{~cm}^{2}\right)$. This was followed by transferring the piece of the skin to the Franz cell which was filled with $\mathrm{pH} 6.8$ phosphate buffer to equilibration for around $30 \mathrm{~min}$. Then, a $2.5-\mathrm{cm}^{2}$ portion of the transdermal patch was positioned on the diffusion cell, with the help of O-ring and glass disk assembly, and finally secured with the clamp. The reservoir compartment contained $5.0 \mathrm{~mL}$ of phosphate buffer, $\mathrm{pH} 6.8$, from which $0.3 \mathrm{~mL}$ was withdrawn periodically using autosampler and analyzed by validated HPLC method. The cumulative medium correction was made to determine the total amount of lidocaine permeated at each time points. This experiment was repeated with three skin donors, and three diffusion cells were used at each time $(n=3) . \% C t_{\mathrm{n}}$ was calculated using the following Eq. (1):

$\% C t_{\mathrm{n}}=\frac{C_{\mathrm{t}} \times V \times 100}{S \times C_{\mathrm{T}}}$

where $\% C t_{\mathrm{n}}=$ normalized value of cumulative percent drug permeated, $C_{\mathrm{t}}=$ concentration at time $t, V=$ volume of dissolution medium, $S=$ surface area of skin, and $C_{T}=$ concentration at terminal time point.

\section{In Vivo Studies}

Human Volunteers. All the pharmacokinetic studies were approved by the Institutional Review Boards (IRBs) and were conducted in accordance with the Declaration of Helsinki and Good Clinical Practice Guidelines. In study I, 18 human volunteers were enrolled, and in study II, 36 human volunteers were enrolled. For both the studies, study population included healthy, non-smoking, non-drinking males and females (nonpregnant) between the ages of 18 to 45 years and with a body mass index of $18-30 \mathrm{~kg} / \mathrm{m}^{2}$. Subjects were screened by medical history, clinical laboratory tests, and physical and skin examination. Absence of pregnancy was evaluated by urine pregnancy test. Subjects not meeting the above said criteria were excluded from the study. Vital signs such as temperature, pulse rate, and blood pressure were assessed prior to each treatment 
and also after 6 and $12 \mathrm{~h}$ of the patch application. Throughout the duration of both the studies, volunteers were continuously observed and questioned for the occurrence of any adverse events. Written consent was obtained from all participants prior to entry into the study.

\section{Studies I and II}

Studies I and II were performed in an open-label, single-center, and randomized environment. LTP is a $10 \times$ $14 \mathrm{~cm}$ patch that contains $5 \%$ lidocaine within its adhesive layer, and each subject received three patches for application. All the transdermal patches were applied to clean, dry, non-oily, non-irritated, and non-recently shaved skin, on the lower/mid-back area. The application area selected was away from any significant fold/creases and at least 1 in away from the spine.

Blood samples of $7 \mathrm{~mL}$ were collected periodically at -1 , $0,1.5,3,4,6,8,9,10,11,12,13,14,15,16,20,24,30$, and $36 \mathrm{~h}$ of post-patch-application, and plasma drug concentrations were determined by a validated liquid chromatography-tandem mass spectrometry (LC-MS/MS) method. General adhesion of the patches was analyzed at 2, 4, 6, 8, 10, and $12 \mathrm{~h}$ after application. The subjects with poor adhesion score were excluded from estimation of plasma drug concentration and PK analyses.

\section{Bioanalytical Method for Estimation of Lidocaine}

Blood samples were centrifuged and the plasma stored at $-20^{\circ} \mathrm{C}$ until analysis by LC-MS/MS. The preparation of column and the quantification of lidocaine were similar to those mentioned in literature with slight modifications (20, 21). Lidocaine- $d_{10}$ was used as internal standard (IS). Lidocaine plasma concentrations were analyzed by fortifying a $50-\mu \mathrm{L}$ sample aliquot with $20 \mu \mathrm{L}$ of internal standard working solution. A $200-\mu \mathrm{L}$ solution of $2.0 \%$ ammonium hydroxide was then added, vortexed, and centrifuged. Then, the organic layer was transferred to a clean tube and a $20-\mu \mathrm{L}$ volume of this final extract was injected and analyzed via HPLC equipped with MS/MS detection. The intraday and interday precision and accuracy are found to be within $15 \%$ from both percent coefficient of variation $(\% \mathrm{CV})$ and percent difference from theoretical. The limit of quantification was found to be $0.2 \mathrm{ng} / \mathrm{mL}$. The average recovery (\%) of drug and IS was 95.4 and 99.6, respectively. Stability of QC samples in plasma during normal handling was determined to be acceptable after stressing with four freeze-thaw cycles and room temperature exposure for $24 \mathrm{~h}$. Stability of the drug for $24 \mathrm{~h}$ in extracts was also acceptable. No interfering peaks were noted in blank plasma samples.

\section{PK Analyses}

From both studies, PK data of LTP was taken for further analysis. PK analysis for both studies was done by using Phoenix software version 6.3 (Pharsight $^{\mathrm{TM}}$, Certara L.P.). The PK parameters such as peak plasma concentration $\left(C_{\max }\right)$, time to reach $C_{\max }\left(T_{\max }\right)$, area under plasma concentration time curve from time zero to time of last measurable concentration $\left(A U C_{0-\mathrm{t}}\right)$, area under plasma concentration time curve from time zero to time infinity $\left(A U C_{0-\text { inf }}\right)$, and terminal elimination half-life $\left(t_{1 / 2}\right)$ were calculated for both studies.

\section{PK Modeling}

PK modeling of lidocaine was performed by using plasma drug concentration-time profile of intravenous bolus (50 $\mathrm{mg}$ dose) data obtained from the literature (4). Compartmental analysis was performed by using Phoenix version 6.3 (Pharsight $^{\mathrm{TM}}$, Certara L.P.). PK Solver was used for non-compartmental analysis (22). Selection of the model was based on best fit approach and other statistical parameters.

\section{IVIVC}

Level A correlation was estimated by a two-stage procedure: deconvolution followed by comparison of fraction drug absorbed in vivo $\left(F_{\mathrm{a}}\right)$ to the fraction of drug permeated in vitro $\left(F_{\mathrm{p}}\right)$. Percent in vitro permeated was calculated by Eq. (1). Percent in vivo absorbed profile was calculated by using NCA and NDC methods.

In NDC method, IV data which follows a twocompartment pharmacokinetic model was used to calculate the unit impulse response (UIR) values. The obtained UIR values were used to deconvolute the plasma drug concentration-time profiles of LTP. NDC method analysis was done by using Phoenix version 6.4 (Pharsight $^{\mathrm{TM}}$, Certara L.P). Equation (2) represents the percent in vivo permeation of the drug using numerical deconvolution method:

$C(t)=\int_{o}^{t} C_{\delta}(t-u) r_{\mathrm{abs}}(u) d u$

where $r_{\text {abs }}$ is absorption rate time course, $C_{\delta}$ represents the concentration-time profile resulting from an instantaneous absorption of a unit amount of drug which is typically from bolus intravenous injection or reference oral solution data, $C(t)$ represents the plasma concentration versus time profiles of the tested formulations, and $u$ is the variable of integration.

In NCA method, the percent in vivo permeated was estimated by using the following Eq. (3):

$\%$ in vivo absorbed $=\frac{A U C_{(0-\mathrm{t})} \times K_{\mathrm{el}} \times V d}{F \times D} \times 100$

where $A U C_{(0-\mathrm{t})}=$ area under time curve from time point $(0$ $t), K_{\mathrm{el}}=$ elimination constant, $V_{\mathrm{d}}=$ volume of distribution, $F=$ percent bioavailable from the formulation, and $D=$ dose administered.

The internal validation for the developed IVIVC by NCA method has been carried out by comparing the observed values versus predicted values from the same study, whereas external validation was performed by using predicted values of study I versus observed values of study II and vice versa. 
The percent prediction error ( $\% \mathrm{PE})$ was calculated by using the Eq. (4):

$\% P E=\frac{\text { Observed Value }- \text { Predicted value }}{\text { Observed value }} \times 100$

\section{RESULTS AND DISCUSSION}

\section{Ex Vivo Skin Permeation Studies}

The results of ex vivo skin permeation of lidocaine from LTP are shown in Fig. 1a. The average cumulative amount $(\mu \mathrm{g})$ and percent $(\%)$ of drug permeated from the LTP in $24 \mathrm{~h}$ were $205.89 \pm 52.31$ and $6.43 \pm 1.6$, respectively. However, the percent in vivo absorption of lidocaine from patches was reported as $3 \pm 2 \%$ (8). The differences between ex vivo and in vivo skin permeation could be due to various reasons such as skin preparation methods, viability of the skin, age of the skin, area of harvesting, and analytical method parameters such as surface area, drug loading, and volume of the media. The run-to-run variation in average cumulative permeation due to the above mentioned factors is rate limiting for establishing IVIVC, and hence $\% C t_{\mathrm{n}}$ was calculated to overcome the variation. The comparative statistical analysis of $\% C t_{\mathrm{n}}$ in three different studies ( $n=3$ for each study) at all the time points, except first time point, shows less variability when compared to percent cumulative release. The calculated RSD (\%) was found to be 16 and 58 for $\% C t_{\mathrm{n}}$ and percent

a

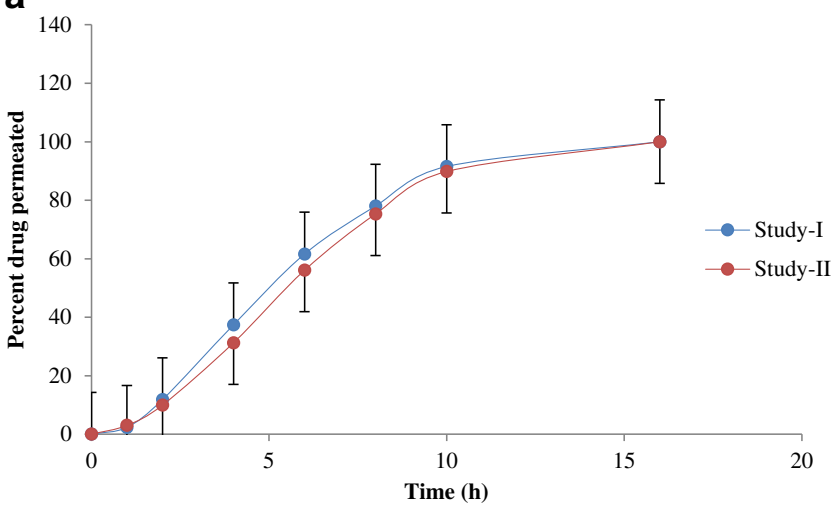

b

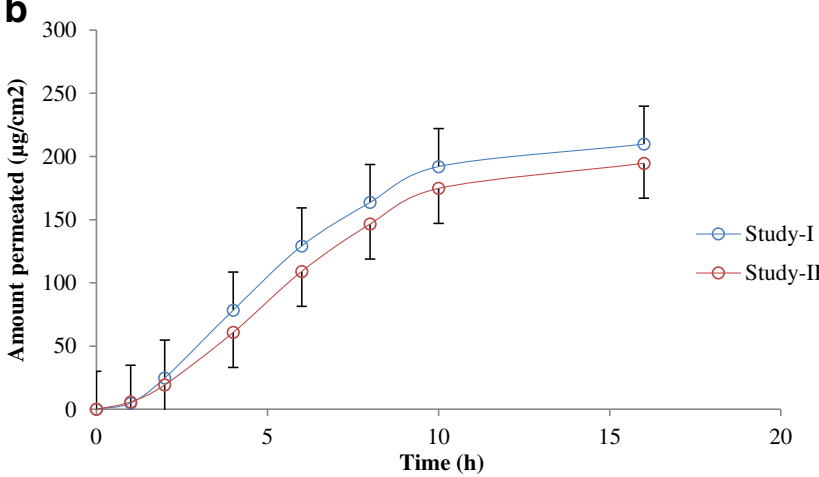

Fig. 1. a In vitro skin permeation profiles for study I and study II. Percent lidocaine permeated. Data are presented as mean $\pm \operatorname{SEM}(n=$ 3). b In vitro skin permeation profiles for study I and study II. Amount lidocaine permeated. Data are presented as mean $\pm \operatorname{SEM}(n=3)$ cumulative release, respectively. Figure $1 \mathrm{~b}$ represents the $\% C t_{\mathrm{n}}$ of LTP. The release kinetics of the drug from drug delivery system was computed with respect to main mathematical models such as Higuchi's kinetics, zero-order kinetics, first-order kinetics, and polynomial method. The goodness of fit for various models investigated for Lidoderm topical patches ranked in the following order:

polynomial $>$ first-order $>$ zero-order $>$ Higuchi's kinetics.

All the kinetic models, other than the polynomial, did not fit at the initial time points. Table I summarizes the statistical analysis of various mathematical models. Polynomial model was chosen for establishing IVIVC and to calculate the average flux.

\section{In Vivo Study}

The PK parameters of studies I and II are summarized in Table II. Figure 2 depicts the plasma concentration profiles for both the studies. Out of 18 volunteers, 17 volunteers completed the study I. Mean $C_{\max }, A U C_{0-\mathrm{t}}$, and $A U C_{0 \text {-inf }}$ were found to be $39.7 \mathrm{ng} / \mathrm{mL}(\mathrm{CV}=54.6 \%), 568.1 \mathrm{ng} \cdot \mathrm{h} / \mathrm{mL}(\mathrm{CV}=49.7 \%)$, and $590.8 \mathrm{ng} \cdot \mathrm{h} / \mathrm{mL}(\mathrm{CV}=48.7 \%)$, respectively. A total of 35 volunteers (out of 36) completed the study II. Mean values for $C_{\text {max }}, A U C_{0-\mathrm{t}}$, and $A U C_{0 \text {-inf }}$ were found to be $38.5 \mathrm{ng} / \mathrm{mL}$ $(\mathrm{CV}=23 \%), 564.3 \mathrm{ng} \cdot \mathrm{h} / \mathrm{mL}(\mathrm{CV}=11.1 \%)$, and $582.6 \mathrm{ng} \cdot \mathrm{h} / \mathrm{mL}$ $(\mathrm{CV}=9.6 \%)$, respectively.

\section{Pharmacokinetic Modeling}

Pharmacokinetic modeling plays an integral part in the establishment of IVIVC (23). Pharmacokinetic parameters

Table I. In Vitro Release Kinetics of Both Study I and Study II

\begin{tabular}{|c|c|c|c|c|}
\hline \multirow[t]{2}{*}{ Parameter } & \multicolumn{2}{|c|}{ Amount permeated } & \multicolumn{2}{|c|}{ Percent permeated } \\
\hline & Study I & Study II & Study I & Study II \\
\hline \multicolumn{5}{|l|}{ Higuchi } \\
\hline Observed $r^{2}$ & 0.9590 & 0.959 & 0.959 & 0.9559 \\
\hline Adjusted $r^{2}$ & 0.8758 & 0.8612 & 0.8758 & 0.8612 \\
\hline $\mathrm{AIC}$ & 71.96 & 71.50 & 60.11 & 60.84 \\
\hline \multicolumn{5}{|l|}{ First-order } \\
\hline Observed $r^{2}$ & 0.8869 & 0.8947 & 0.9847 & 0.9811 \\
\hline Adjusted $r^{2}$ & 0.4344 & 0.5312 & 0.9370 & 0.9268 \\
\hline AIC & 84.09 & 81.23 & 54.68 & 55.71 \\
\hline \multicolumn{5}{|l|}{ Second-order } \\
\hline Observed $r^{2}$ & 0.9452 & 0.9559 & 0.9452 & 0.9556 \\
\hline Adjusted $r^{2}$ & 0.8834 & 0.9093 & 0.8834 & 0.9093 \\
\hline AIC & 71.45 & 68.09 & 59.60 & 57.00 \\
\hline \multicolumn{5}{|l|}{ Zero-order } \\
\hline Observed $r^{2}$ & 0.9452 & 0.9556 & 0.9469 & 0.9556 \\
\hline Adjusted $r^{2}$ & 0.8834 & 0.9093 & 0.8872 & 0.9093 \\
\hline AIC & 59.60 & 57.4 & 59.71 & 57.44 \\
\hline \multicolumn{5}{|l|}{ Polynomial } \\
\hline Observed $r^{2}$ & 0.995 & 0.998 & 0.9971 & 0.998 \\
\hline Adjusted $r^{2}$ & 0.992 & 0.996 & 0.995 & 0.996 \\
\hline AIC & - & - & - & - \\
\hline
\end{tabular}

AIC Akaike information criterion 
Table II. Comparative Analysis of PK Modeling

\begin{tabular}{|c|c|c|c|c|c|c|c|}
\hline \multirow[t]{2}{*}{ Parameter } & \multicolumn{3}{|l|}{ IV } & \multicolumn{2}{|l|}{ Study I } & \multicolumn{2}{|l|}{ Study II } \\
\hline & NCA & One-compartment & Two-compartment & NCA & One-compartment & NCA & One-compartment \\
\hline$C_{0}(\mathrm{ng} / \mathrm{mL})$ & 5944.20 & 5312.05 & 6002.22 & - & - & - & - \\
\hline$C_{\max }(\mathrm{ng} / \mathrm{mL})$ & 5415.21 & & - & $39.71 \pm 23.38$ & 28.53 & $38.56 \pm 16.24$ & 25.59 \\
\hline $\begin{array}{l}A U C_{0-\mathrm{t}} \\
\quad(\mathrm{ng} \cdot \mathrm{h} / \mathrm{mL})\end{array}$ & 2680.53 & 1165.19 & 2617.41 & $568.01 \pm 280.67$ & 702.07 & $564.31 \pm 204.34$ & 712.84 \\
\hline$t^{1 / 2}(\mathrm{~h})$ & 1.4 & 0.152 & $\begin{array}{l}\text { Slow: } 0.072 \\
\text { Fast: } 1.27\end{array}$ & 6.0 & $\begin{array}{l}t_{1 / 2 \mathrm{a}}: 7.07 \\
t_{1 / 2 \mathrm{e}}: 7.66\end{array}$ & 5.7 & $\begin{array}{l}t_{1 / 2 \mathrm{a}}: 6.61 \\
t_{1 / 2 \mathrm{e}}: 7.58\end{array}$ \\
\hline $\mathrm{A}(\mathrm{ng} / \mathrm{mL})$ & - & - & 4698.51 & - & 989.08 & - & 585.63 \\
\hline Alpha (1/h) & - & - & 9.60 & - & 0.098 & - & 0.091 \\
\hline B (ng/mL) & - & - & 1303.71 & - & & - & - \\
\hline Beta $(1 / \mathrm{h})$ & - & - & 0.54 & - & & - & - \\
\hline $\mathrm{AIC}$ & - & 163.50 & 116.50 & - & 125.38 & - & 128.30 \\
\hline Adjusted $r^{2}$ & & 0.96 & 0.99 & - & 0.93 & - & 0.92 \\
\hline
\end{tabular}

$t_{1 / 2 \mathrm{a}}$ absorption half-life, $t_{1 / 2 \mathrm{e}}$ elimination half-life, Slow from central compartment to peripheral compartment, Fast peripheral compartment to central compartment, AIC Akaike information criterion, $N C A$ non-compartmental analysis

were obtained by using non-compartmental analyses (NCA). The obtained parameters such as $A U C_{0-\mathrm{t}}, C_{\max }, T_{\max }$, and $t_{1 / 2}$ cannot be used directly in a compartment model, and their transformation to compartment model PK parameters is essential. Compartmental (one-compartment and two-compartment) PK parameters, such as absorption rate constant $\left(K_{\mathrm{a}}\right)$, elimination rate constant $\left(K_{\mathrm{el}}\right)$, and volume of distribution $\left(V_{\mathrm{d}}\right)$, were obtained using non-compartmental parameters such as $A U C_{0-\mathrm{t}}, T_{\max }$, and $t_{1 / 2}$ (24). One-compartment and two-compartment open models are frequently used for deconvolution of the plasma drug concentration time profile to obtain percent in vivo drug absorption. However, the prediction error associated with one-compartment and twocompartment models affects the quality of obtained deconvoluted data. Intravenous PK data obtained from the literature (4) was used for estimation of percent in vivo absorbed from NDC method. Based on the AIC values and $r^{2}$ provided in Table II, the two-compartmental open model was selected as the best fitting PK model for lidocaine. Twocompartmental and non-compartmental PK parameters were used for establishing IVIVC by NDC and NCA methods, respectively. Table II summarizes the comparative statistical

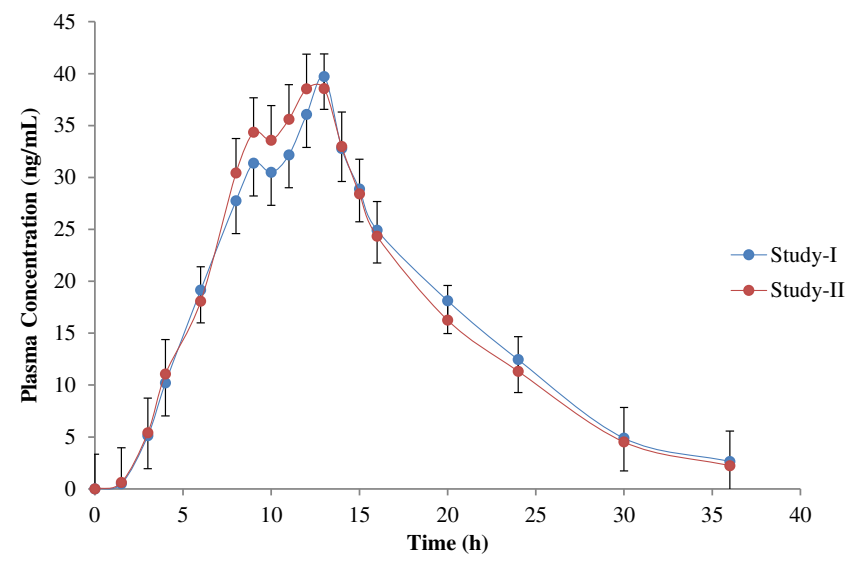

Fig. 2. PK profiles of study I and study II. Data are presented as mean \pm SEM ( $n=17$ for study I; $n=35$ for study II) analysis of lidocaine administered through IV and extravascular (transdermal) route of administration.

\section{IVIVC}

Level A correlation between normalized percent cumulative in vitro permeation and in vivo permeation was established using non-compartmental analysis (NCA) and numerical deconvolution (NDC) method. The presence of test
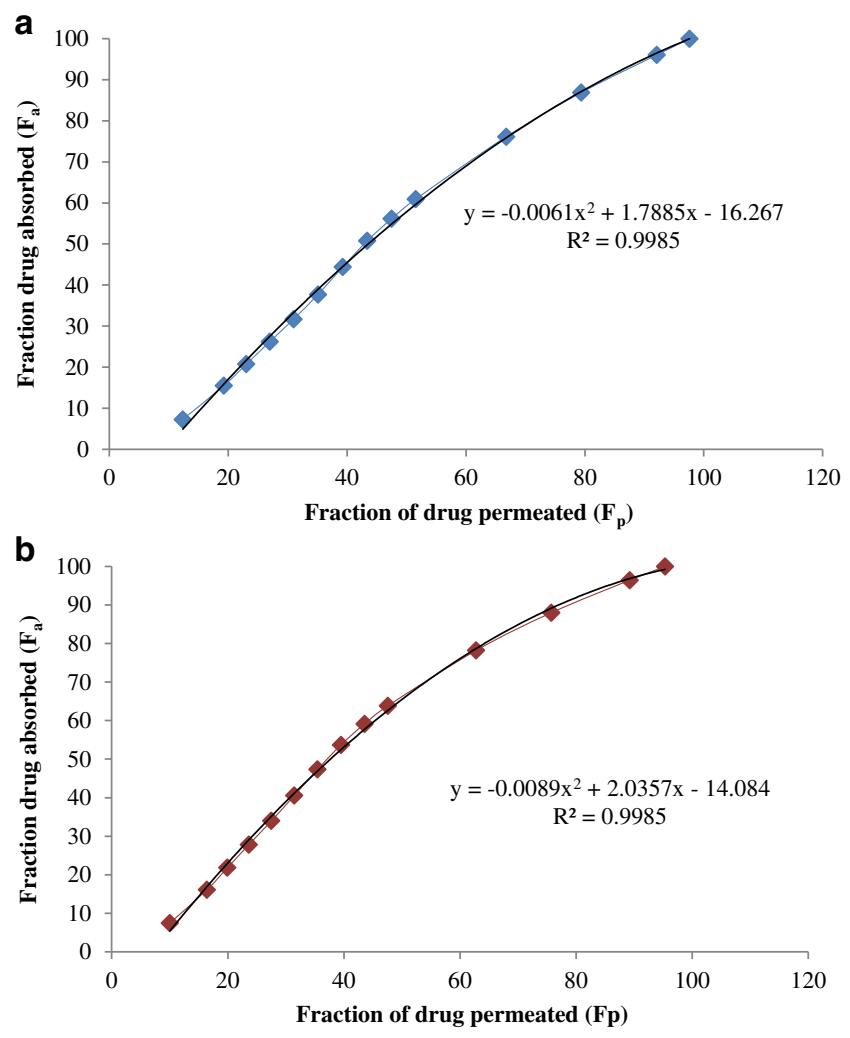

Fig. 3. a Fraction of drug permeated in vitro and in vivo for study I. b Fraction of drug permeated in vitro and in vivo for study II 

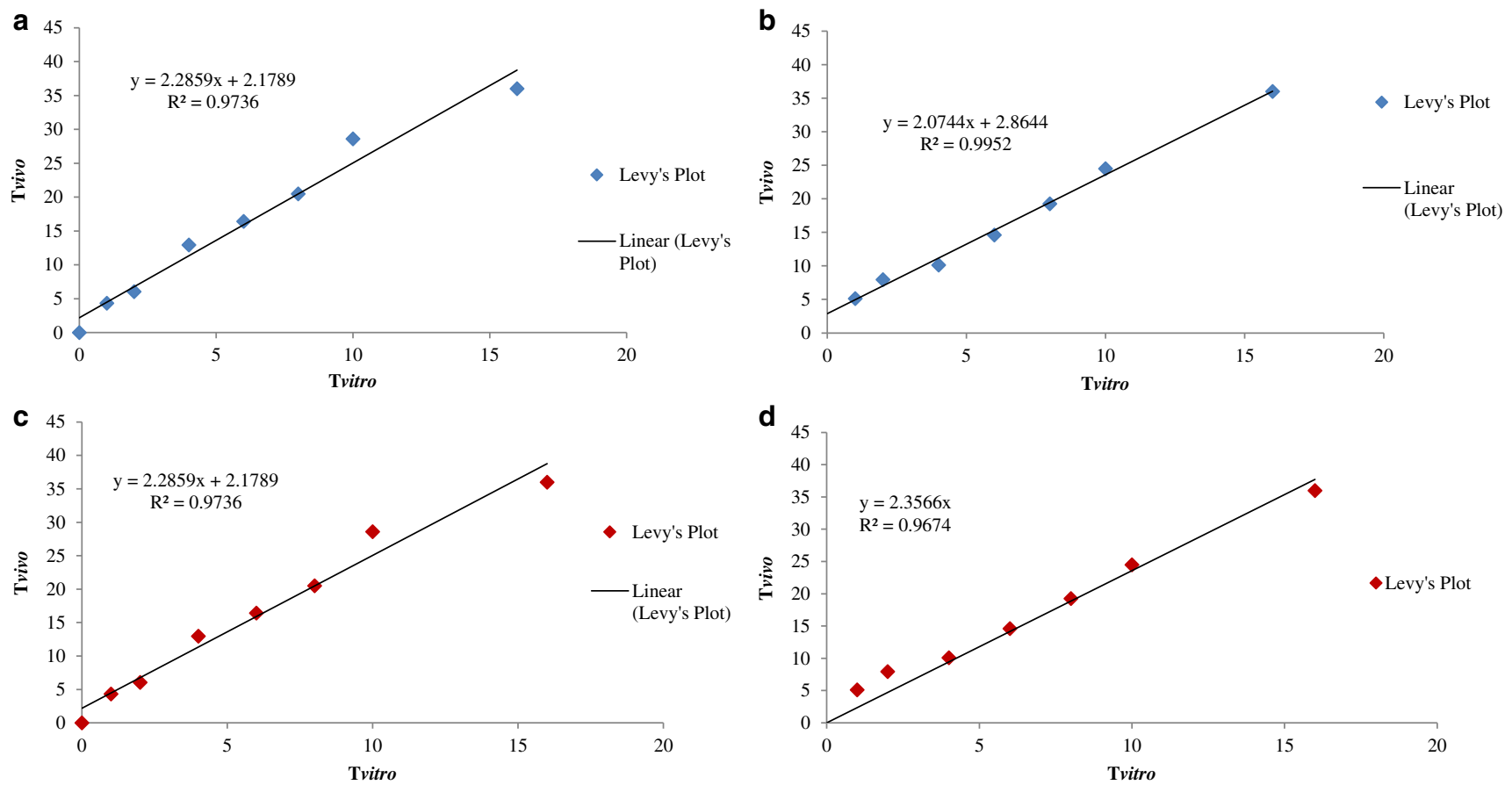

Fig. 4. a Levy's plot of NCA for study I. b Levy's plot of NCA for study II. c Levy's plot of NCA of study I (external validation). d Levy's plot of NCA of study II (external validation)

a

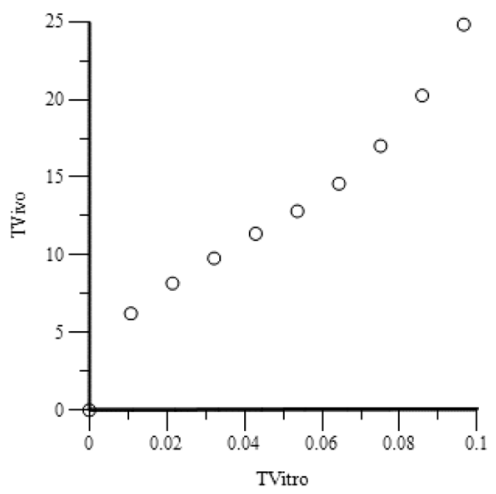

C Rsq $_{\mathrm{q}}=0.9599$, Intercept $=2.1$, Slope $=277.5$

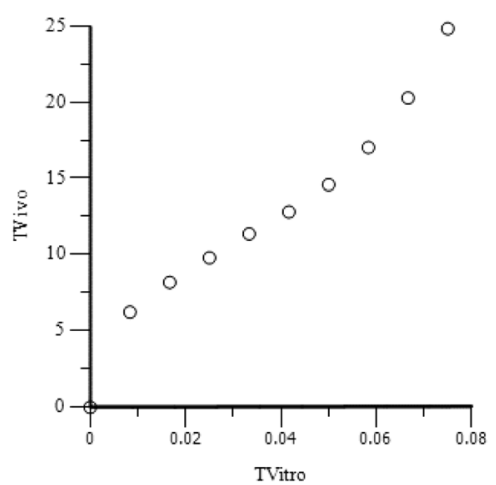

b

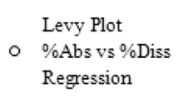

- Unity Line

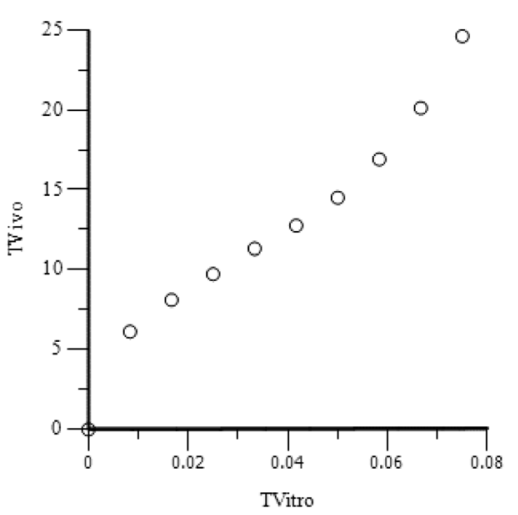

d

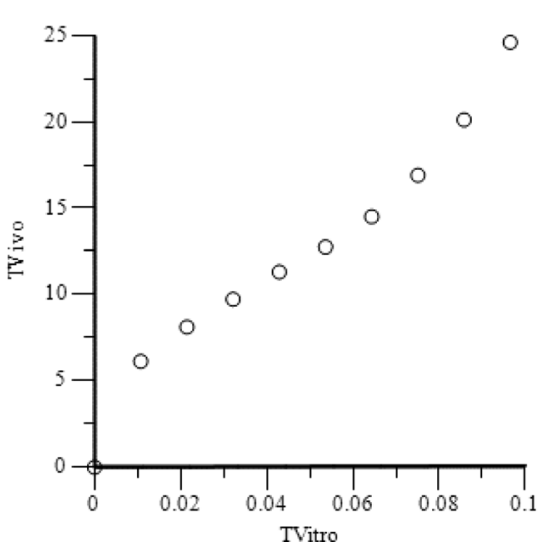

Levy Plot

- \%Abs vs $\%$ Diss

Regression

- Unity Line

Fig. 5. a Levy's plot of NDC for study I. b Levy's plot of NDC for study II. c Levy's plot of NDC for study I (external validation). d Levy's plot of NDC for study II (external validation) 

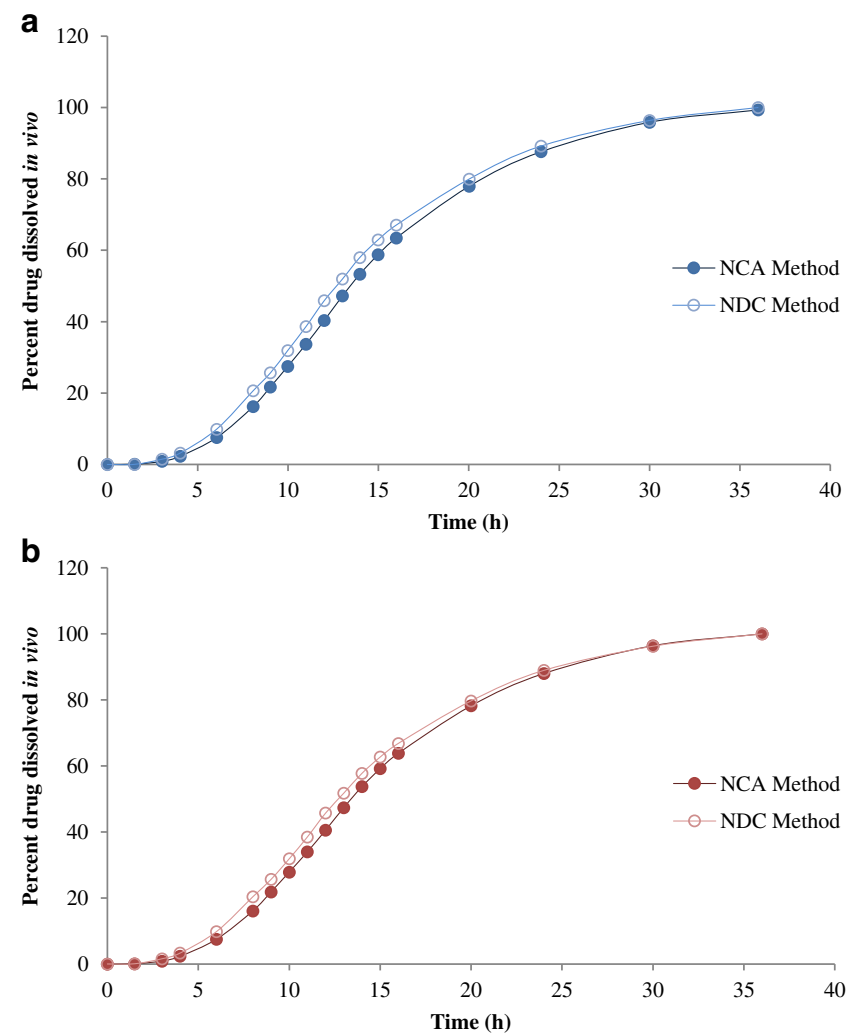

Fig. 6. a Comparison for percent in vivo drug release of study I from both NCA and NDC methods. b Comparison for percent in vivo drug release of study II from both NCA and NDC methods

formulation is usually required for the development of IVIVC, in order to understand and validate the above method; in vitro permeation and in vivo absorption data of RLD product only has been utilized in the current study. The percent (\%) in vivo permeated was predicted by non-compartmental analysis of PK data obtained from study I and study II using Eq. (3). The main objective of using NCA was to reduce the residuals/ errors during the modeling. Moreover, the dose normalization was done using the cumulative amount drug in the body at 36 h. Figure $3 \mathrm{a}, \mathrm{b}$ shows the relationship between $F_{\mathrm{p}}$ and $F_{\mathrm{a}}$. Various mathematical models were evaluated and the best fitting model was selected based on statistical parameters. The best fitting mathematical model for studies I and II were $y=-0.0061 x^{2}+1.7885 x-16.267$ and $y=-0.0089 x^{2}+2.0357 x-$ 14.084, respectively. The PK parameters such as $C_{\max }$ and $A U C_{\text {last }}$ were predicted with the above established equations. Figure 4a, b shows Levy's plots for study I and study II obtained between in vitro time and in vivo time with a correlation coefficient $\left(r^{2}\right)$ of 0.9977 and 0.9976 , respectively. The sensitivity of the established IVIVC methods was evaluated by constructing the Levy's plots between in vitro study I versus in vivo study II and in vitro study II versus in vivo study I and is depicted from Fig. 4c, d, respectively. The slope and regression values obtained from Levy's plots indicated the robustness of the developed mathematical models for both studies (I and II). The advantages of this IVIVC method over conventional methods are (a) minimal compartmental modeling error, (b) easy to calculate in vivo permeation, and (c) simple mathematical expressions. Unlike Loo-Riegelman method, IV data is not required to calculate the unit impulse response (UIR) for deconvolution process.

Using Phoenix ${ }^{\circledR}$ (version 6.4), IVIVC was established with numerical deconvolution method. Figure 5a, b shows Levy's plots of study I and study II with $r^{2}$ values of 0.9976 and 0.995 , respectively. The internal validation for established IVIVCs was carried out by using the established mathematical expression, whereas external validation was done using flipping of the mathematical expressions between studies I and II. Figure 5c, d shows the externally validated Levy's plot for both study I and study II.

The comparison of percent in vivo release for both NCA and NDC methods is illustrated in Fig. 6a, b, respectively. Table III summarizes the predicted PK values and associated errors of NCA and NDC methods for both the studies (I and II). The percent prediction error of $C_{\max }$ in study I, using internal validation method, was found to be -30.9 and $3.9 \%$ for NDC and NCA methods, respectively. The high prediction error of $C_{\max }$ in NDC might be due to two-compartment PK modeling error for both studies I and II. The direct deconvolution of plasma drug concentration with time profile using NCA analysis resulted in low prediction error, especially for internal validation. Moreover, the external validation results obtained from the NCA and NDC methods for studies I and II confirm the comparability of NCA method over NDC method. A prediction error of less than $2 \%$ in both studies of NCA and NDC methods imparts the robustness of NCA method in line with NDC method.

Table III. Validation and Prediction of PK Parameters

\begin{tabular}{|c|c|c|c|c|c|c|c|c|}
\hline \multirow[t]{2}{*}{ Study } & \multirow[t]{2}{*}{ PK model } & \multirow[t]{2}{*}{ Type of validation } & \multicolumn{2}{|l|}{ Observed } & \multicolumn{2}{|c|}{ Predicted } & \multicolumn{2}{|c|}{$\%$ Prediction error } \\
\hline & & & $C_{\max }$ & $A U C_{\text {last }}$ & $C_{\max }$ & $A U C_{\text {last }}$ & $C_{\max }$ & $A U C_{\text {last }}$ \\
\hline \multirow[t]{4}{*}{ Study I } & \multirow[t]{2}{*}{ NDC } & Internal & \multirow[t]{4}{*}{$39.71 \pm 23.38$} & \multirow[t]{4}{*}{$568.01 \pm 280.67$} & 27.40 & 569.06 & -30.9 & 0.1 \\
\hline & & External & & & 30.60 & 578.07 & 22.9 & 1.7 \\
\hline & \multirow[t]{2}{*}{ NCA } & Internal & & & 41.30 & 579.69 & 3.9 & 2.0 \\
\hline & & External & & & 50.68 & 579.19 & 27.6 & 1.9 \\
\hline \multirow[t]{4}{*}{ Study II } & \multirow[t]{2}{*}{ NDC } & Internal & \multirow[t]{4}{*}{$38.56 \pm 16.24$} & \multirow[t]{4}{*}{$564.31 \pm 204.34$} & 29.38 & 562.05 & -23.8 & 0.4 \\
\hline & & External & & & 28.76 & 564.60 & -25.4 & 0.1 \\
\hline & \multirow[t]{2}{*}{ NCA } & Internal & & & 45.9 & 563.35 & 16.1 & -0.2 \\
\hline & & External & & & 38.57 & 553.0 & 0.03 & 2.0 \\
\hline
\end{tabular}


The objective of the present study was to correlate in vitro and in vivo skin permeation profiles of lidocaine in its transdermal patches. Additionally establishing the bioequivalence between RLD and test products is a tedious process. The objectives of the present study can be better achieved if the bioequivalence is achieved between an inhouse developed transdermal patch of lidocaine and an RLD. However, as a preliminary study, we used two separate batches of RLD product to study the correlation of in vitro and in vivo skin permeation profiles and then to establish bioequivalence between two different batches. We wanted to establish pharmacokinetic modeling and in vitro-in vivo correlation (IVIVC) for different batches of RLD and then to adopt the same principles for the developed (test) product.

Moreover, there are no reports available on the establishment of IVIVC for commercialized transdermal patch (15). The transdermal patch like Lidoderm ${ }^{\circledR}$ has poor bioavailability $(3,8)$ which is considered as a bottleneck for developing IVIVC. In the present study, we attempted to establish a preliminary IVIVC method by NCA method to demonstrate the correlation between in vitro and in vivo skin permeation of lidocaine. IVIVC was also established using NDC method, but the mathematical expression obtained from NDC method was used to evaluate the IVIVC obtained from NCA method. Both NDC and NCA methods provided a good correlation between in vitro and in vivo drug permeation across the skin; however, the NCA method was found to be superior to the NDC method. Since, $C_{\max }$ is one of the critical PK parameters for establishing bioequivalence, and hence, both methods were used to predict the $C_{\max }$ values. The predicted $C_{\max }$ values for NCA method were found to be closer to the observed value, when compared with $C_{\max }$ values obtained from NDC method. This further confirms the superiority of NCA method in establishing IVIVC over NDC method in this study.

\section{CONCLUSION}

The present study successfully develops the correlation between in vitro and in vivo skin permeation of lidocaine in its transdermal patch. Based on the results of the present study, the NCA method is a superior approach over NDC method in predicting the $C_{\mathrm{max}}$ and $A U C_{\text {last }}$ after administration of LTP. The NCA method would be most useful if the drug has longer absorption half-life and shorter elimination half-life. The study successfully shows that NCA method may be used as a biorelevant quality control (BRQC) in vitro tool for quality assurance of transdermal products. However, detailed studies are required to validate the results obtained from this study.

\section{ACKNOWLEDGMENTS}

The authors would like to thank Dr. Sreenivasa Vanapalli, Senior Principal Scientist, Clinical PK/PD, for his critical opinion about this article. The authors would also like to thank B. Madhava Rao, Senior Scientist, for his support in pharmacokinetic analyses and Dr. Reddy's Laboratories, Hyderabad, India, for providing facilities and financial assistance to publish this work.

\section{Conflict of interest None}

\section{REFERENCES}

1. Prausnitz MR, Langer R. Transdermal drug delivery. Nat Biotechnol. 2008;26(11):1261-8. doi:10.1038/nbt.1504.

2. Galer BS, Rowbotham MC, Perander J, Friedman E. Topical lidocaine patch relieves postherpetic neuralgia more effectively than a vehicle topical patch: results of an enriched enrollment study. Pain. 1999;80(3):533-8.

3. Campbell BJ, Rowbotham M, Davies PS, Jacob 3rd P, Benowitz NL. Systemic absorption of topical lidocaine in normal volunteers, patients with post-herpetic neuralgia, and patients with acute herpes zoster. J Pharm Sci. 2002;91(5):1343-50. doi:10.1002/jps.10133.

4. Collinsworth KA, Kalman SM, Harrison DC. The clinical pharmacology of lidocaine as an antiarrhythymic drug. Circulation. 1974;50(6):1217-30. doi:10.1161/01.cir.50.6.1217.

5. Krumova EK, Zeller M, Westermann A, Maier C. Lidocaine patch $(5 \%)$ produces a selective, but incomplete block of $\mathrm{A} \delta$ and C fibers. Pain. 2012;153(2):273-80. doi:10.1016/ j.pain.2011.08.020.

6. Response to Citizen Petition 2006. http://www.fda.gov/ohrms/ dockets/dockets/06p0522/06p-0522-cp00001-01-vol1.pdf. Accessed 17.07.2015.

7. FDA Guidance Documents US Food and Drug Administration. 2015. http://www.fda.gov/RegulatoryInformation/Guidances/. Accessed 17.07.2015.

8. Public Assessment Report- Lidocaine 5\% medicated plaster MHRA. 2015. http://www.mhra.gov.uk/home/groups/par/documents/websiteresources/con2032998.pdf. Accessed 17.07.2015.

9. Dissolution methods FDA. 2007. http://www.accessdata.fda.gov/ scripts/cder/dissolution/dsp_SearchResults_Dissolutions.cfm. Accessed 17/07/2007.

10. Venter JP, Muller DG, du Plessis J, Goosen C. A comparative study of an in situ adapted diffusion cell and an in vitro Franz diffusion cell method for transdermal absorption of doxylamine. Eur J Pharm Sci. 2001;13(2):169-77.

11. Sun L, Cun D, Yuan B, Cui H, Xi H, Mu L, et al. Formulation and in vitro/in vivo correlation of a drug-in-adhesive transdermal patch containing azasetron. J Pharm Sci. 2012;101(12):4540-8. doi:10.1002/jps.23317.

12. Liu J, Wang Z, Liu C, Xi H, Li C, Chen Y, et al. Silicone adhesive, a better matrix for tolterodine patches-a research based on in vitro/in vivo studies. Drug Dev Ind Pharm. 2012;38(8):1008-14. doi:10.3109/03639045.2011.637049.

13. Yang Z, Teng Y, Wang H, Hou H. Enhancement of skin permeation of bufalin by limonene via reservoir type transdermal patch: formulation design and biopharmaceutical evaluation. Int $\mathrm{J}$ Pharm. 2013;447(1-2):231-40. doi:10.1016/j.ijpharm.2013.02.048.

14. Khire A, Vavia P. Bioavailability, bioequivalence, and in vitroin vivo correlation of oxybutynin transdermal patch in rabbits. Drug Deliv Transl Res. 2014;4(2):105-15. doi:10.1007/s13346013-0170-y.

15. Van Buskirk GA, Arsulowicz D, Basu P, Block L, Cai B, Cleary $\mathrm{GW}$, et al. Passive transdermal systems whitepaper incorporating current chemistry, manufacturing and controls (CMC) development principles. AAPS PharmSciTech. 2012;13(1):218-30. doi:10.1208/s12249-011-9740-9.

16. Emami J. In vitro - in vivo correlation: from theory to applications. J Pharm Pharm Sci. 2006;9(2):169-89.

17. Pentikis H, Henderson J, Tran N, Ludden T. Bioequivalence: individual and population compartmental modeling compared to the noncompartmental approach. Pharm Res. 1996;13(7):1116-21. doi:10.1023/A:1016083429903.

18. Roy S, Flynn G. Transdermal delivery of narcotic analgesics: comparative permeabilities of narcotic analgesics through human cadaver skin. Pharm Res. 1989;6(10):825-32. doi:10.1023/ A:1015944018555.

19. Singh I, Morris AP. Performance of transdermal therapeutic systems: effects of biological factors. Int J Pharm Investig. 2011;1(1):4-9. doi:10.4103/2230-973X.76721. 
20. Aarons L. Applied Biopharmaceutics and Pharmacokinetics, leon shargel and Andrew B. C. Yu. J Clin Pharm Ther. 1981;6(4):287-8. doi:10.1111/j.1365-2710.1981.tb01006.x.

21. A sensitive method for the determination of lidocaine in human plasma by LC-MS-MS Cedra Corporation. 2007. http:// www.wwctrials.com/uploads/tx_wctpostersandpresentations/ 2007.11.26___Ljungqvist_-_Lidocaine.pdf. Accessed 17.07.2015.

22. Zhang Y, Huo M, Zhou J, Xie S. PKSolver: an add-in program for pharmacokinetic and pharmacodynamic data analysis in
Microsoft Excel. Comput Methods Prog Biomed. 2010;99(3):306-14. doi:10.1016/j.cmpb.2010.01.007.

23. Modi NB, Lam A, Lindemulder E, Wang B, Gupta SK. Application of in vitro-in vivo correlations (IVIVC) in setting formulation release specifications. Biopharm Drug Dispos. 2000;21(8):321-6.

24. Wang Z, Kim S, Quinney S, Zhou J, Li L. Non-compartment model to compartment model pharmacokinetics transformation meta-analysis - a multivariate nonlinear mixed model. BMC Syst Biol. 2010;4 Suppl 1:1-7. doi:10.1186/1752-0509-4-S1-S8. 